\title{
Erratum: Mechanism for quantum speedup in open quantum systems [Phys. Rev. A 93, 020105(R) (2016)]
}

Hai-Bin Liu, W. L. Yang, Jun-Hong An $\odot$, and Zhen-Yu Xu

(Received 30 July 2021; published 31 August 2021)

DOI: 10.1103/PhysRevA.104.029907

In our original article, the labels for the two lines in Fig. 1(b) were accidentally interchanged. The correct form of Fig. 1 is as follows.
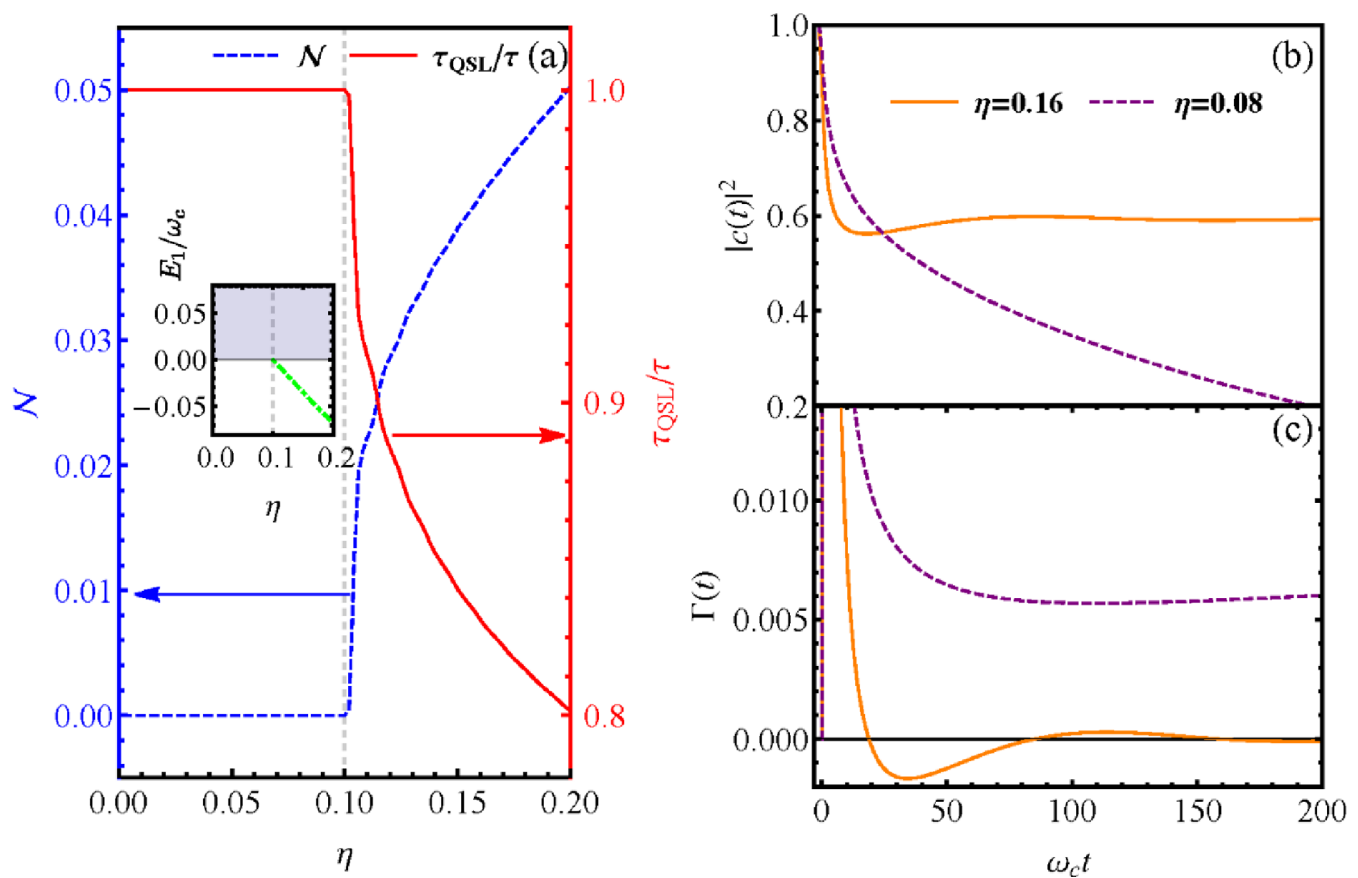

FIG. 1. (a) Non-Markovianity $\mathcal{N}$ (blue dashed line) and QSL time $\tau_{\mathrm{QSL}}$ (red solid line) as a function of the coupling constant $\eta$. The inset shows the energy spectrum of the total system, where the green dot-dashed line denotes the energy of the formed bound state. The obtained $|c(t)|^{2}$ (b) and the corresponding decay rate $\Gamma(t)$ (c) with and without bound state. The bound state is formed when $\eta>\eta_{c}=0.1$. The parameters are $\omega_{c} \tau=800, \omega_{0} / \omega_{c}=0.1$, and $s=1$. 